\title{
ERRATUM
}

\section{Kimiko Terai \\ Parties with policy preferences and uncertainty over voter behavior}

Published online: 20 May 2006

(C) Springer-Verlag 2006

\section{Soc Choice Welfare (2006) DOI 10.1007/s00355-006-0120-4}

Unfortunately a few author corrections were not carried out. The correct mathematical terms are shown below:

In the second line after Eq. (5)

$$
\begin{aligned}
& Y\left(x, \beta_{i}\right) \\
& e^{-\frac{1}{1+\underline{\beta}}}<x_{L}<m<x_{R}<e^{-\frac{1}{1+\bar{\beta}}}
\end{aligned}
$$

\section{Assumption 1}

In the fourth line above Eq. (8)

$$
Y\left(x, \beta_{i}\right)>Y\left(x, \beta_{j}\right)
$$

In the second line above Eq. (8)

$$
Y\left(x, \beta_{i}\right)>Y\left(x, \beta_{j}\right)
$$

The online version of the original article can be found at http://dx.doi.org/10.1007/s00355-006$0120-4$ 